\title{
CARACTERIZACIÓN GENÉTICA DEL CABALLO MONCHINO Y SU RELACIÓN CON OTRAS RAZAS AUTÓCTONAS ESPAÑOLAS
}

\author{
GENETIC CHARACTERIZATION OF THE CABALLO MONCHINO BREED AND ITS \\ RELATIONSHIPS WITH OTHER SPANISH LOCAL EQUINE BREEDS
}

Tupac-Yupanqui, I. ${ }^{1}$, Dunner, S. ${ }^{1}$, Sañudo, B. ${ }^{2}$, González, A. ${ }^{2}$, Argüello, S. de ${ }^{2}$, Barquín, F. ${ }^{2}$, Crespo, M.J. ${ }^{2}$, Chomón, N. ${ }^{2}$, Cimadevilla, C. ${ }^{2}$, Calderón, L.A. ${ }^{2}$, Fernández, L.A. ${ }^{3}$ y Cañón, J. ${ }^{1 *}$

1'Departamento de Producción Animal. Facultad de Veterinaria. Madrid. España. *jcanon@vet.ucm.es ${ }^{2}$ Consejería de Desarrollo Rural, Ganadería, Pesca y Biodiversidad. Santander. España.

${ }^{3}$ Asociación Regional de Criadores de Ganado Equino de Raza Monchina. Santander. España.

\section{Palabras clave adicionales}

Diversidad genética. Microsatélites.

\section{RESUMEN}

Para la caracterización genética de una población de Caballo Monchino se ha utilizado la información proporcionada por 12 marcadores del tipo microsatélite. Además se utilizaron 481 muestras de otras 7 razas autóctonas y se calcularon los parámetros de diversidad genética poblacional, los estadísticos $\mathrm{F}$ de Wright $\left(\mathrm{F}_{\mathrm{IS}} \mathrm{F}_{\mathrm{ST}}\right)$ y un análisis de correspondencia. Se puede concluir que se trata de una población significativamente diferenciada.

\section{SUMMARY}

In this study 12 microsatellite markers are used to characterize the Caballo Monchino horse breed and to known the genetic relationships with other Spanish local horse breeds. The genetic diversity, allelic frequencies, heterozygosity, Wright's $F$ statistics (FIS and FST) was calculated, and to establish the genetics relationship between Mediterranean horse populations a correspondence analysis was carried out.

\section{INTRODUCCIÓN}

La aparición del Caballo Monchino se remonta a la prehistoria, así lo demuestra la existencia de pinturas en las cuevas de Altamira en la Comunidad de Cantabria. Sobre su origen existen diversas versiones

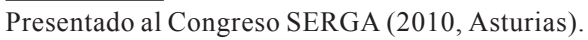

\section{AdDitional KEYWORDS}

Genetic diversity. Microsatellites.

que señalan una supuesta procedencia de la fusión entre el tronco del caballo cantábrico y el tronco castellano, siendo éste último el más importante. La zona de origen se concentra en los montes más nororientales de la Comunidad de Cantabria (Ayuntamientos de Guriezo, Rasines y Castro-Urdiales), zonas especialmente montañosas donde se cría en condiciones de libertad. Al ser un animal semisalvaje, el movimiento de las manadas es constante entre toda el área noroeste de Cantabria, expandiéndose esporádicamente a los ayuntamientos de Trucios y Carranza en Vizcaya.

Es un animal de gran rusticidad, elipométrico, subcóncavo y brevilineo o subrevilineo con un peso en torno a los 260$330 \mathrm{~kg}$ y una alzada entre 1,35 y 1,46 m. Presenta el cuello corto y robusto, orejas pequeñas y muy móviles, tronco desarrollado, pecho amplio, costillares ligeramente arqueados, espalda alargada y con tendencia a la oblicuidad; dorso ligeramente ensillado, lomo corto, fuerte y bien unido, vientre con frecuencia voluminoso. Extremidades finas con articulaciones fuertes, sistema vascular desarrollado y bien definido, cascos pequeños, duros y negros, en oca- 
Tabla I. Principales parámetros genéticos poblacionales del Caballo Monchino. (Results of the main population genetic parameters for the Monchino local horse breed).

\begin{tabular}{lr}
\hline Número efectivo de alelos & 4,42 \\
Heterocigosis esperada & 0,748 \\
Heterocigosis observada & 0,737 \\
Probabilidad de coincidencia & $2,6 \times 10^{13}$ \\
Probabilidad de exclusión & \\
$\quad$ ambos padres conocidos & 99,995 \\
$\quad$ 1 sólo padre conocido & 99,688 \\
$\mathrm{~F}_{\text {Is }}$ & 0,0283 \\
\hline
\end{tabular}

siones veteados. Su capa es color negra azabache o castaña, siempre con crines y colas negras; es frecuente la presencia de lucero, cordón corrido o estrella en la frente, se admite calzado hasta la mitad de la caña.

E1 25 de Mayo de 2000 se creó el Libro de Registro Genealógico de la Raza Equina Monchina (Orden, de 25 de mayo de 2000); en la actualidad el número de explotaciones en el área de origen es de $25 \mathrm{y}$ el censo total es de 700 animales identificados.

Una de las principales causas de diferenciación genética entre poblaciones de una misma especie, es la deriva genética, sobre todo si aquellas tienen un número de

Tabla II. Distancia genética $\left(F_{S T}\right)$ de cada una de las razas con respecto al resto de razas, y valores de heterocigosis observada y esperada. (Genetic distance $\left(\mathrm{F}_{\mathrm{ST}}\right)$ of each of the breeds to the rest, and observed and expected heterozygosities).

\begin{tabular}{lcccc}
\hline Raza & Número & $\mathrm{F}_{\mathrm{ST}}$ & $\mathrm{Ho}$ & $\mathrm{He}$ \\
\hline Asturcón & 119 & 0,071 & 0,739 & 0,729 \\
JacaNava & 122 & 0,063 & 0,703 & 0,728 \\
Losino & 66 & 0,077 & 0,719 & 0,702 \\
Poni Gallego & 72 & 0,042 & 0,716 & 0,76 \\
Pottoka & 51 & 0,043 & 0,742 & 0,77 \\
Menorquina & 31 & 0,070 & 0,75 & 0,726 \\
Mallorquina & 20 & 0,087 & 0,708 & 0,748 \\
Monchino & 50 & 0,049 & 0,737 & 0,748 \\
\hline
\end{tabular}

reproductores reducido y existe aislamiento reproductivo entre ellas. A este propósito, y cuando la información genealógica es escasa o no existe, se considera que la mejor forma de reflejar las posibles diferencias consecuencia del aislamiento genético es mediante la utilización de marcadores de tipo microsatélite que en principio son neutros tanto a la selección natural, como a la selección artificial.

El objetivo del estudio fue la caracterización genética del Caballo Monchino utilizando información molecular genéticamente neutra.

\section{MATERIAL Y MÉTODOS}

Se han utilizado 12 marcadores del tipo microsatélite: AHT4, AHT5, ASB2, HMS2, HMS3, HMS6, HMS7, HTG4, HTG6, HTG7, HTG10, VHL20 en 50 muestras de sangre. Para establecer la posición relativa de esta raza hemos utilizado la información de otras 7 razas mediterráneas (Asturcón, Jaca Navarra, Losino, Poni Gallego, Pottoka, Menorquí, Mallorquí) con un total de 481 muestras. Para el análisis estadístico se han utilizado programas estándar como MICROSATELLITE TOOLKIT (Park, 2001), MEGA (Tamura et al., 2007) y GENETIX 4.0 (Belkhir et al., 2001), y se calcularon para cada raza las heterocigosis observada Ho y esperada He (tabla I), así como los estadísticos de F Wright's (Wright, 1965) $\mathrm{F}_{\mathrm{ST}}$ y $\mathrm{F}_{\text {IS }}$ para analizar la distribución de la variabilidad genética entre razas.

\section{RESULTADOSYDISCUSIÓN}

Se obtuvieron los principales parámetros genéticos poblacionales del Caballo Monchino (tabla I). Podemos observar que las probabilidades de coincidencia $l a$ probabilidad de que dos individuos tomados al azar de la raza tengan el mismo genotipo es suficientemente reducida como para que se pueda garantizar la trazabilidad

Archivos de zootecnia vol. 60, núm. 231, p. 426. 


\section{CARACTERIZACIÓN GENÉTICADEL CABALLOMONCHINO}

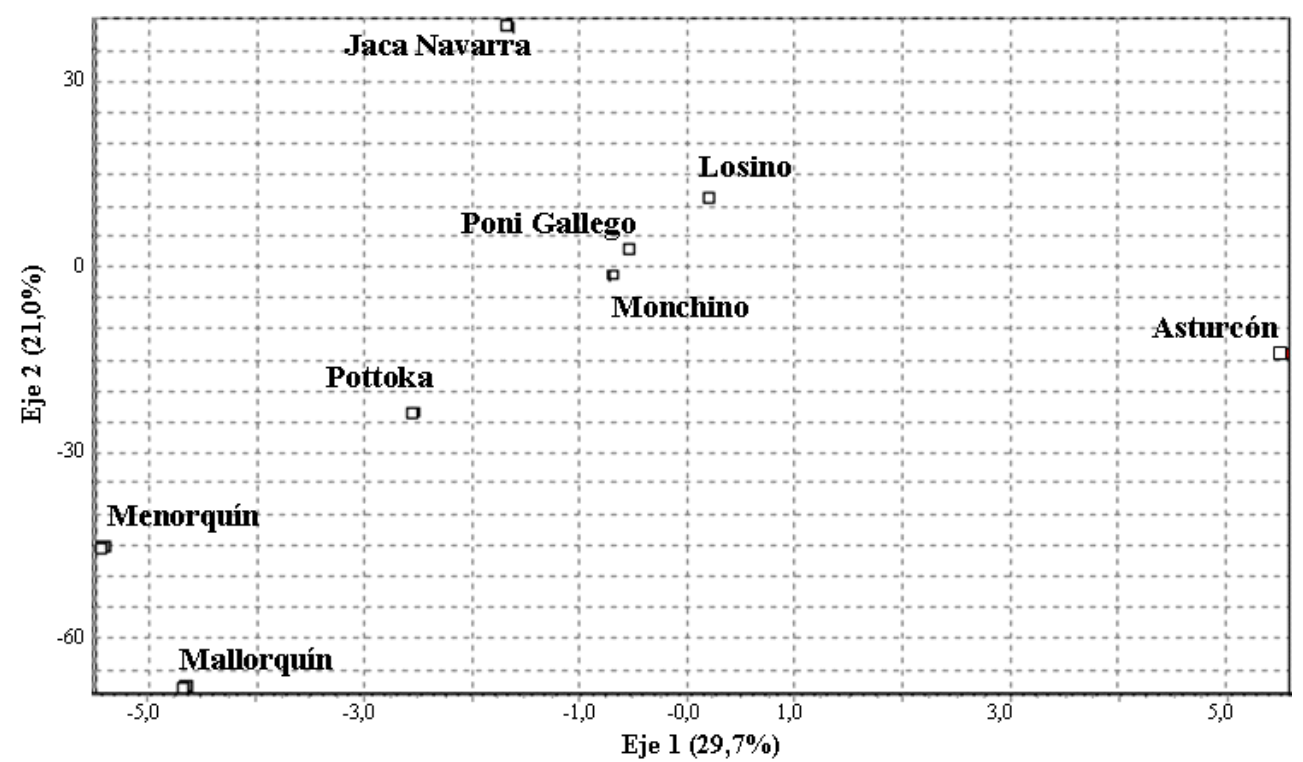

Figura 2. Representación gráfica del análisis de correspondencia utilizando la información de 12 marcadores de tipo microsatellite. (Graphic representation of the correspondence analysis which used the information provided by 12 microsatellites).

individual utilizando este conjunto de marcadores. Algo similar se puede decir de las potencias de exclusión de paternidad, incluso cuando disponemos de un solo padre. El valor que se obtiene en el caso de disponer de un solo padre es del 99,7 y en todo caso superior al $99 \%$ cuando se consideran ambos padres.
Se estimó la distancia genética $\mathrm{F}_{\mathrm{ST}}$ de cada una de las razas con respecto al resto de razas y puede observarse la posición del Caballo Monchino (tabla II). También se presentan los resultados de diversidad génica, heterocigosis esperada y observada. La distancia de la población del Caballo Monchino con respecto a las otras pobla-

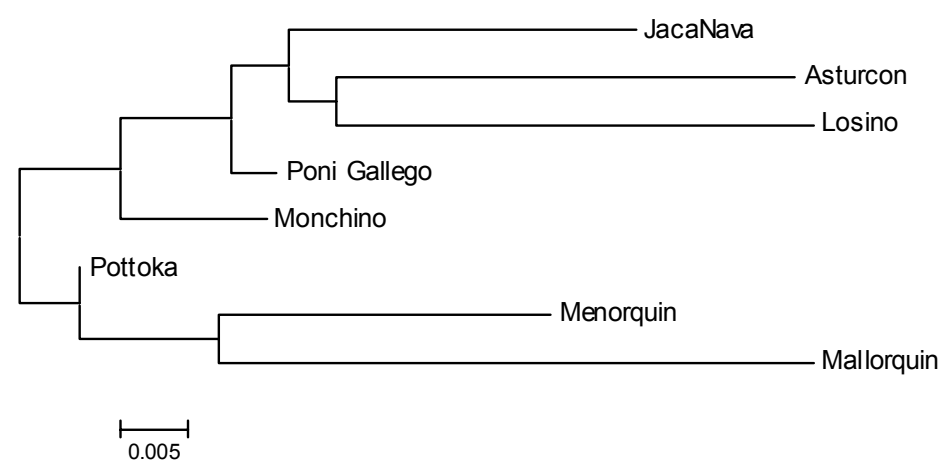

Figura 1. Dendrograma mostrando la relación genética entre razas de caballos. (Dendrogram showing the genetic relationships hmong the local equine breeds). 
ciones fue de un 5\%. Los niveles de diferenciación entre razas $\left(\mathrm{F}_{\mathrm{ST}}\right.$ total) indican que alrededor del $6 \%$ de la diferencia se debe a las diferencias entre razas y el $94 \%$ a la diferencia entre los individuos.

El valor del $\mathrm{F}_{\mathrm{ST}}$ entre las poblaciones parece ser consecuencia de las distancias entre las razas atlánticas y las razas mediterráneas. La distancia media entre las razas atlánticas fue del 5\% y entre las mediterráneas del $8 \%$, resultados que coinciden con otros previamente publicados (Cañon et al., 2000).

El valor obtenido de $\mathrm{F}_{\text {IS }}$ en el Caballo Monchino fue 0,028 (tabla I), y el de $\mathrm{F}_{\mathrm{ST}}$ fue de 0,049 , valor diferente de 0 que muestra el grado de aislamiento reproductivo respecto al conjunto de razas incluidas en el análisis. Por otro lado, estos resultados están dentro del rango de los publicados previamente (Cañón et al., 2000; Marletta et al., 2006).

Con el fin de ver la posición relativa del Caballo Monchino con otras poblaciones de caballos con los que ha podido compartir ancestros, se incluyó en el análi-

\section{BIBLIOGRAFÍA}

Belkhir, K., Borsa, P., Chikhi, L., Raufaste, N. et Bonhomme, F. 2001. Genetix, logiciel sous Windows TM pour la génétique des populations, laboratoire génome, populations, interactions. CNRS UPR 9060. Université de Montpellier II. Montpellier. France. Available online: http://www. genetix.univ-montp2.fr/genetix/intro.htm (10/02/ 10).

Cañón J., Checa, M.L., Carleos, C., Vega-Pla, J.L., Vallejo, M. and Dunner, S. 2000. The genetic structure of Spanish Celtic horse breeds inferred from microsatellite data. Anim. Genet., 31: 39-48.

Falconer, D. S. and Mackay, T.F.C. 1996. Introduction to quantitative genetics, $4^{\mathrm{a}}$ ed. Longmans Green. Harlow. Essex. UK.

Marletta, D., Tupac-Yupanqui, I., Bordonaro, S., García, D., Guastella, A., Criscione, A., Cañon, J. and Dunner, S. 2006. Analysis of genetic diversity and the determination of relationships sis un conjunto de razas de caballos mediterráneos de los que se disponía de información genética similar a la utilizada en los análisis del Caballo Monchino. La representación gráfica de las distancias entre parejas de poblaciones (figura 1), destaca la proximidad de la población del Caballo Monchino respecto al Caballo Gallego, esta proximidad relativa es la que también se deduce de la representación en dos dimensiones de la posición que ocupan las diferentes razas cuando los ejes (dimensiones) son una combinación de la información molecular utilizada en un análisis de correspondencia (figura 2).

Los resultados genéticos obtenidos muestran una raza relativamente homogénea, como indica su reducido valor de $\mathrm{F}_{\text {IS }}$, con valores de parámetros genéticos dentro del rango del que expresan las razas equinas autóctonas españolas, y que aparece significativamente diferenciada de ellas como muestra el valor del $\mathrm{F}_{\mathrm{ST}}$. El conjunto de marcadores microsatélites permitirá una trazabilidad suficientemente potente, así como una adecuada gestión genealógica.

among western Mediterranean horse breeds using microsatellite markers. J. Anim. Breed Genet., 123: 315-325.

Orden de 25 de mayo de 2000, por la que se crea el libro de registro genealógico de la raza equina Monchina y se aprueba su funcionamiento. Boletín Oficial de Cantabria, núm 111, de 8 de junio de 2000.

Park, S.D.E. 2001. Trypanotolerance in West African Cattle and the population genetic effects of selection. Ph.D. Thesis. University of Dublin. Dublin. Ireland.

Tamura, K., Dudley, J., Nei, M. and Kumar, S. 2007. MEGA4: Molecular evolutionary genetics analysis (MEGA) software version 4.0. Mol. Biol. Evol., 24: 1596-1599.

Wright, S. 1965. The interpretation of population structure by F-statistics with special regard to systems of mating. Evolution, 19: 395-420. 